\title{
Bifurcating solutions in a constrained minimization problem of elasticity
}

\author{
Adair R. Aguiar ${ }^{1}$ \\ EESC/USP, São Carlos, SP \\ Lucas A. Rocha ${ }^{2}$ \\ EESC/USP, São Carlos, SP
}

\begin{abstract}
We consider the problem of a cylindrically anisotropic disk subject to an imposed displacement on its boundary. In the context of classical linear elasticity, the solution of this problem is not locally injective. This characterizes material overlapping, which is not physically admissible. To prevent this anomalous behavior, we minimize the energy functional of classical linear elasticity subject to the local injectivity constraint. One possible solution of the associated Euler-Lagrange equations, reported in the literature, is radially symmetric. In this work we search for a secondary solution, which is rotationally symmetric. In the region where the constraint is not active, we determine closed-form expressions for the displacement field. In the region where the constraint is active, which is annular, we determine a relation between the components of the displacement field to ensure the imposition of this constraint. The expressions obtained in both regions depend on constants of integration that are determined numerically. In addition, we also determine the inner and outer radii of the active annular region. This research is of interest in the investigation of solids with radial microstructure, such as carbon fibers.
\end{abstract}

Keywords. Anisotropic disk, Elasticity, Constrained minimization, Local injectivity, Penalty method, Finite element method

\section{Introduction}

There are problems in classical linear elasticity whose closed-form solutions, while satisfying the governing equations of equilibrium and well-posed boundary conditions, predict material overlapping, which, of course, is not physically realistic. Mathematically, material overlapping is characterized by the loss of injectivity of the deformation field. Locally, it means that the determinant of the deformation gradient is not strictly positive.

In this work, we consider the problem of a homogeneous cylindrically anisotropic disk subject to an imposed displacement on its boundary in the absence of body forces. It is known [5] that, in the context of classical linear elasticity, the solution of this problem predicts material overlapping when the elastic modulus in the radial direction is greater than the elastic modulus in the tangential direction, which is a material property found in carbon fibers with radial microstructure [3].

To prevent this anomalous behavior, Fosdick and Royer-Carfagni [5] proposed to minimize the classical energy functional of classical linear elasticity subject to the local injectivity constraint, which consists of imposing that the determinant of deformation gradient be no less that a arbitrarily small positive parameter. They show that under suitable, but otherwise sufficiently general

\footnotetext{
1 aguiarar@sc.usp.br.

2 lucas.almeida.rocha@usp.br.
} 
boundary conditions, a solution exists. The authors considered the disk problem mentioned above to derive a closed-form solution that is locally injective and radially symmetric, in the sense that the displacement field has only the radial component and depends only on the radius.

The associated constrained minimization problem has a constraint that is highly nonlinear and active in an unknown region. This poses difficulties to determine closed-form solutions without imposing a priori assumption on symmetry. Alternatively, Aguiar [1] proposed a numerical procedure based on an interior penalty formulation together with the finite element method that does not require a priori knowledge of the region where the constraint is active.

Fosdick, Freddi and Royer-Carfagni [4] revisited the constrained disk problem and, using the numerical procedure proposed in [1], searched for a solution with no a priori assumption on symmetry. Their computational results show that there is a secondary solution that seems to be rotationally symmetric, in the sense that its has radial and tangential components that depend only on the radius, and bifurcates from the radially symmetric solution obtained in [5].

In our previous work [2], we searched computationally for a rotationally symmetric solution of the constrained disk problem. We obtained convergent sequences of solutions that tend to a limit function that is different from the computational results presented in [4]. In particular, in [2], the tangential displacement is linear with respect to the radius in a neighborhood of the origin, which is not presented in [4].

In this work, we use the Euler-Lagrange equations of the associated constrained minimization problem to determine closed-form expressions for a secondary solution, which is rotationally symmetric. The expressions are valid in the region where the constraint is not active and depend on constants of integration that are determined numerically. In the region where the constraint is active, which is annular, we determine a nonlinear relation between the radial and tangential components of the solution. This relation also depends on a constant of integration that is determined numerically. In addition, we also determine the inner and outer radii of the annular active region. In spite of some differences mentioned above, the results presented in this work are in good agreement with the computational results obtained in [2].

\section{The disk problem}

Let $\mathcal{B} \subset \mathbb{R}^{2}$ be the undistorted natural reference configuration of a linearly elastic body. Points $\mathbf{X} \in \mathcal{B}$ are mapped to points $\mathbf{x}:=\mathbf{f}(\mathbf{X})=\mathbf{X}+\mathbf{u}(\mathbf{X}), \mathbf{x} \in \mathbb{R}^{2}$, where $\mathbf{u}(\mathbf{X})$ is the displacement of $\mathbf{X}$. The boundary $\partial \mathcal{B}$ of $\mathcal{B}$ is composed of two non-intersecting parts, $\partial_{1} \mathcal{B}$ and $\partial_{2} \mathcal{B}, \partial \mathcal{B}=\partial_{1} \mathcal{B} \cup \partial_{2} \mathcal{B}$, $\partial_{1} \mathcal{B} \cap \partial_{2} \mathcal{B}=\emptyset$, such that $\mathbf{u}(\mathbf{X})=\overline{\mathbf{u}}(\mathbf{X})$ for $\mathbf{X} \in \partial_{1} \mathcal{B}$, where $\overline{\mathbf{u}}$ is a given function, and a dead load traction field $\overline{\mathbf{t}}(\mathbf{X})$ is prescribed for $\mathbf{X} \in \partial_{2} \mathcal{B}$. In addition, a body force $\mathbf{b}(\mathbf{X})$ per unit of volume acts on $\mathbf{X} \in \mathcal{B}$.

In the constrained minimization theory [5], we consider the minimization problem

$$
\min _{\mathbf{u} \in \mathcal{A}_{\varepsilon}} \mathcal{E}[\mathbf{u}], \quad \mathcal{E}[\mathbf{u}]=\frac{1}{2} \int_{\mathcal{B}} \mathbb{C}[\mathbf{E}] \cdot \mathbf{E} d \mathbf{X}-\int_{\mathcal{B}} \mathbf{b} \cdot \mathbf{u} d \mathbf{X}-\int_{\partial_{2} \mathcal{B}} \overline{\mathbf{t}} \cdot \mathbf{u} d \mathbf{X},
$$

where

$$
\mathcal{A}_{\varepsilon}:=\left\{\mathbf{u} \in \mathcal{W}^{1,2}(\mathcal{B}) \rightarrow \mathbb{R}^{2} \mid \operatorname{det}(\mathbf{1}+\nabla \mathbf{u}) \geq \varepsilon>0, \mathbf{u}=\overline{\mathbf{u}} \text { on } \partial_{1} \mathcal{B}\right\}
$$

is the set of kinematically admissible solutions with $\varepsilon$ being a sufficiently small positive parameter. In (1), $\mathbb{C}$ is the elasticity tensor, which is symmetric and positive definite, and $\mathbf{E}$ is the infinitesimal strain tensor given by

$$
\mathbf{E}=\left[\nabla \mathbf{u}+(\nabla \mathbf{u})^{T}\right] / 2 .
$$

Let $\mathcal{B}=\mathcal{B}_{>} \cup \mathcal{B}_{=}, \mathcal{B}_{>} \cap \mathcal{B}_{=}=\emptyset$, where

$$
\mathcal{B}_{>}:=\operatorname{int}[\{\mathbf{X} \in \mathcal{B} \mid \operatorname{det} \nabla \mathbf{f}(\mathbf{X})>\varepsilon\}], \quad \mathcal{B}_{=}:=\operatorname{int}[\{\mathbf{X} \in \mathcal{B} \mid \operatorname{det} \nabla \mathbf{f}(\mathbf{X})=\varepsilon\}],
$$


where int[·] denotes the interior of a set. The Euler-Lagrange equations for the constrained minimization problem defined by (1)-(2) are given by

$$
\operatorname{Div} \mathbf{T}+\mathbf{b}=\mathbf{0} \quad \text { in } \mathcal{B}_{>}, \quad \operatorname{Div}\left(\mathbf{T}-\varepsilon \lambda(\nabla \mathbf{f})^{-T}\right)+\mathbf{b}=\mathbf{0} \quad \text { in } \mathcal{B}_{=},
$$

where $\lambda(\mathbf{X}) \geq 0$ is the Lagrange multiplier field associated with the injectivity constraint $\operatorname{det}(\mathbf{1}+$ $\nabla \mathbf{u}) \geq \varepsilon>0$ and $\mathbf{T}=\mathbb{C}[\mathbf{E}]$ is the stress tensor. The boundary conditions are given by

$$
\mathbf{T} \mathbf{n}=\overline{\mathbf{t}} \quad \text { in } \partial_{2} \mathcal{B}_{>}, \quad\left(\mathbf{T}-\varepsilon \lambda(\nabla \mathbf{f})^{-T}\right) \mathbf{n}=\overline{\mathbf{t}} \quad \text { in } \partial_{2} \mathcal{B}_{=}
$$

where $\mathbf{n}$ is a unit normal to $\partial_{2} \mathcal{B}$. In addition, the jump condition

$$
\left.\left(\mathbf{T}-\varepsilon \lambda(\nabla \mathbf{f})^{-T}\right)\right|_{\Sigma \cap \overline{\mathcal{B}}_{=}} \mathbf{n}=\left.\mathbf{T}\right|_{\Sigma \cap \overline{\mathcal{B}}_{>}} \mathbf{n}
$$

must hold across $\Sigma:=\overline{\mathcal{B}}_{>} \cap \overline{\mathcal{B}}_{=}$, where $\mathbf{n}$ is a unit normal to $\Sigma$ and where $\Sigma \cap \overline{\mathcal{B}}_{=}$and $\Sigma \cap \overline{\mathcal{B}}_{>}$mean that the evaluations are understood as limits to the dividing interface $\Sigma$ from within $\mathcal{B}_{=}$and $\mathcal{B}_{>}$, respectively.

In this work, we consider that $\mathcal{B}$ is a circular disk of radius $R_{e}$, with its center coinciding with the origin of the polar coordinate system $(R, \Theta)$, which has an associated orthonormal basis $\left\{\mathbf{e}_{r}, \mathbf{e}_{\theta}\right\}$. In addition, there are no body forces, $\mathbf{b}=\mathbf{0}$, and the entire boundary of the disk is subject to an imposed displacement $\overline{\mathbf{u}}=\bar{u}_{r} \mathbf{e}_{r}, \bar{u}_{r}<0$, yielding $\partial_{2} \mathcal{B}=\emptyset$. The disk is composed of a homogeneous and cylindrically anisotropic material, such that the elasticity tensor $\mathbb{C}$ is constant relative to $\left\{\mathbf{e}_{r}, \mathbf{e}_{\theta}\right\}$.

The stress and strain tensors

$$
\begin{gathered}
\mathbf{T}=\sigma_{r r} \mathbf{e}_{r} \otimes \mathbf{e}_{r}+\sigma_{\theta \theta} \mathbf{e}_{\theta} \otimes \mathbf{e}_{\theta}+\sigma_{r \theta}\left(\mathbf{e}_{r} \otimes \mathbf{e}_{\theta}+\mathbf{e}_{\theta} \otimes \mathbf{e}_{r}\right), \\
\mathbf{E}=\epsilon_{r r} \mathbf{e}_{r} \otimes \mathbf{e}_{r}+\epsilon_{\theta \theta} \mathbf{e}_{\theta} \otimes \mathbf{e}_{\theta}+\epsilon_{r \theta}\left(\mathbf{e}_{r} \otimes \mathbf{e}_{\theta}+\mathbf{e}_{\theta} \otimes \mathbf{e}_{r}\right)
\end{gathered}
$$

are related by the Generalized Hooke's Law, $\mathbf{T}=\mathbb{C}[\mathbf{E}]$, which, here, yields the nonzero components

$$
\sigma_{r r}=c_{11} \epsilon_{r r}+c_{12} \epsilon_{\theta \theta}, \quad \sigma_{\theta \theta}=c_{12} \epsilon_{r r}+c_{22} \epsilon_{\theta \theta}, \quad \sigma_{r \theta}=2 c_{66} \epsilon_{r \theta},
$$

where the elastic moduli $c_{11}, c_{22}, c_{12}$ and $c_{66}$ are constant.

If the constraint $\operatorname{det}(\mathbf{1}+\nabla \mathbf{u})$ was not present in (2), the minimization problem (1)-(2) would become a classical problem of linear elasticity, which has a unique solution $\mathbf{u}: \mathcal{B} \rightarrow \mathbb{R}^{2}$ that is radially symmetric with respect to the center of the disk. This solution is of the form

$$
\mathbf{u}(R, \Theta)=u_{r}(R) \mathbf{e}_{r},
$$

where $u_{r}$ is the radial displacement, which is given by

$$
u_{r}(R)=\bar{u}_{r} \bar{R}^{k},
$$

where $k:=\sqrt{c_{22} / c_{11}}>0$ and $\bar{R}:=R / R_{e}$.

The unconstrained solution (12) yields

$$
\operatorname{det}(\mathbf{1}+\nabla \mathbf{u})=\left(1+k \bar{u}_{r} \bar{R}^{k-1} / R_{e}\right)\left(1+\bar{u}_{r} \bar{R}^{k-1} / R_{e}\right),
$$

which is negative in the interval

$$
-k \bar{u}_{r} / R_{e}<\bar{R}^{1-k}<-\bar{u}_{r} / R_{e}
$$

for $k<1$ and any value of $\bar{u}_{r}<0$. This is a violation of the local injectivity constraint $\operatorname{det}(\mathbf{1}+\nabla \mathbf{u})>$ 0 and represents the existence of regions where the material intersects itself. 
On the other hand, if the constraint $\operatorname{det}(\mathbf{1}+\nabla \mathbf{u})$ is enforced, Fosdick and Royer-Carfagni [5] obtained a solution of the Euler-Lagrange equations (5)-(7) together with a uniform normal pressure on the boundary of the disk that has the form (11). If $k<1$ and recalling from above that, instead of pressure, we are imposing the radial displacement $\bar{u}_{r}$, this solution is given by

$$
u_{r}(R)= \begin{cases}(-1+\sqrt{\varepsilon}) R & \text { for } 0 \leq R \leq R_{c} \\ A R^{k}+B R^{-k} & \text { for } R_{c} \leq R \leq R_{e}\end{cases}
$$

where

$$
A=\frac{1+k}{2 k}(\sqrt{\varepsilon}-1) R_{c}^{-k+1}, \quad B=\frac{-1+k}{2 k}(\sqrt{\varepsilon}-1) R_{c}^{k+1} .
$$

In $(15), R_{c}$ is the radius of the region where the injectivity constraint is active, i.e., where $\operatorname{det}(\mathbf{1}+$ $\nabla \mathbf{u})=\varepsilon$, which is the solution of the equation

$$
(1+k)\left(\frac{R_{e}}{R_{c}}\right)^{k}+(-1+k)\left(\frac{R_{e}}{R_{c}}\right)^{-k}-\frac{2 k \bar{u}_{r}}{(\sqrt{\varepsilon}-1) R_{c}}=0 .
$$

The expressions of the Lagrange multiplier field $\lambda$ and the determinant of the deformation gradient $\operatorname{det}(\mathbf{1}+\nabla \mathbf{u})$ have the same form of the corresponding expressions presented in [5] and will not be presented here.

In this work, we search for a secondary solution of the Euler-Lagrange equations (5)-(7) together with the condition of an imposed radial displacement $\bar{u}_{r}$ on the boundary of the disk that is rotationally symmetric with respect to the center of the disk, in the sense that it has the form

$$
\mathbf{u}(R, \Theta)=u_{r}(R) \mathbf{e}_{r}+u_{\theta}(R) \mathbf{e}_{\theta}
$$

where $u_{r}$ and $u_{\theta}$ are the radial and tangential displacements, respectively. It then follows from (3), (8), (9), (10), (18), and $\mathbf{f}=\mathbf{X}+\mathbf{u}$ that the Euler-Lagrange equations (5)-(7) can be written as

$$
\begin{cases}c_{11} u_{r}^{\prime \prime}+c_{11} \frac{u_{r}^{\prime}}{R}-c_{22} \frac{u_{r}}{R^{2}}=0 & \text { in } \mathcal{B}_{>} \\ u_{\theta}^{\prime \prime}+\frac{u_{\theta}^{\prime}}{R}-\frac{u_{\theta}}{R^{2}}=0 & \end{cases}
$$

and

$$
\begin{cases}c_{11} u_{r}^{\prime \prime}+c_{11} \frac{u_{r}^{\prime}}{R}-c_{22} \frac{u_{r}}{R^{2}}-\lambda^{\prime}\left(1+\frac{u_{r}}{R}\right)=0 & \text { in } \mathcal{B}_{=} . \\ u_{\theta}^{\prime \prime}+\frac{u_{\theta}^{\prime}}{R}-\frac{u_{\theta}}{R^{2}}+\frac{\lambda^{\prime}}{c_{66}+\lambda} u_{\theta}^{\prime}=0 & \end{cases}
$$

Computational results of previous works $[2,4]$ show that $\mathcal{B}_{=}=\left\{\mathbf{X}=R \mathbf{e}_{r} \mid R_{a}<R<R_{b}\right\}$ and $\mathcal{B}_{>}=\mathcal{B}_{>}^{a} \cup \mathcal{B}_{>}^{b}$, where $\mathcal{B}_{>}^{a}:=\left\{\mathbf{X}=R \mathbf{e}_{r} \in \mathcal{B} \mid 0<R<R_{a}\right\}$ and $\mathcal{B}_{>}^{b}:=\left\{\mathbf{X}=R \mathbf{e}_{r} \in \mathcal{B} \mid R_{b}<R<\right.$ $\left.R_{e}\right\}$ for some value of $R_{a}$ and $R_{b}$ such that $0<R_{a}<R_{b}<R_{e}$. Assuming that these expressions hold and imposing the kinematic condition $u_{r}(0)=u_{\theta}(0)=0$, the solution of (19) is given by

$$
\left\{\begin{array}{l}
u_{r}(R)=C_{1} R^{k} \\
u_{\theta}(R)=C_{2} R
\end{array} \quad \text { in } \mathcal{B}_{>}^{a}\right.
$$

and

$$
\left\{\begin{array}{l}
u_{r}(R)=C_{3} R^{k}+C_{4} R^{-k} \\
u_{\theta}(R)=C_{5} R+C_{6} / R
\end{array} \quad \text { in } \mathcal{B}_{>}^{b},\right.
$$


where $C_{i}, i=1,2,3, \ldots, 6$ are constants of integration that are determined below. Note from (21b) that $u_{\theta}(R)$ is linear with respect to $R$, which is in very good agreement with the computational results obtained in our previous work [2]. We then use the boundary conditions $u_{r}\left(R_{e}\right)=\bar{u}_{r}$ and $u_{\theta}\left(R_{e}\right)=0$ in $(22)$ to express $C_{4}$ and $C_{6}$ in terms of $C_{3}$ and $C_{5}$, respectively. Then, we rewrite $(22)$ as

$$
\left\{\begin{array}{l}
u_{r}(R)=C_{3} R^{k}+\left(\bar{u}_{r} R_{e}^{k}-C_{3} R_{e}^{2 k}\right) R^{-k} \\
u_{\theta}(R)=C_{5}\left(R-R_{e}^{2} / R\right)
\end{array} \quad \text { in } \mathcal{B}_{>}^{b} .\right.
$$

In $\mathcal{B}_{=}$, the equations in $(20)$ are complemented by the local injectivity constraint $\operatorname{det}(\mathbf{1}+\nabla \mathbf{u})=$ $\varepsilon$, where $\mathbf{u}$ is given by (18). We then have that this constraint can be written as

$$
g^{\prime}(R)+g(R) / R=2 \varepsilon, \quad g(R):=\left[\left(R+u_{r}\right)^{2}+u_{\theta}^{2}\right] / R,
$$

which has the general solution

$$
g(R)=\varepsilon R+C_{7} / R,
$$

where $C_{7}$ is a constant of integration determined below. It follows from (24b) and (25) that

$$
\left(R+u_{r}\right)^{2}+u_{\theta}^{2}=\varepsilon R^{2}+C_{7} \quad \text { in } \mathcal{B}_{=} .
$$

We still need to solve the nonlinear system of equations consisting of the two equations in (20) and the constraint equation (26) for the three unknown fields $u_{r}, u_{\theta}$, and $\lambda$. In addition, we need to impose continuity conditions on both displacement and traction, given by (7), across the boundaries of the annulus $\mathcal{B}_{=}$. Instead, we use the same numerical procedure of our previous work [2], which is based on an interior penalty formulation and the finite element method proposed in [1], to determine approximate solutions $\mathbf{u}^{N}(R, \Theta)=u_{r}^{N}(R) \mathbf{e}_{r}+u_{\theta}^{N}(R) \mathbf{e}_{\theta}$ of the constrained minimization problem (1)-(2), where $N$ is the number of elements used in the finite element mesh. This numerical procedure also yields approximations of $R_{a}$ and $R_{b}$, which we denote as $R_{a}^{N}$ and $R_{b}^{N}$, respectively.

We use the same material and geometric parameters of [4] in their investigation of the bifurcating solution from the radially symmetric solution (15), that is, $\bar{u}_{r}=-0.05, c_{11}=10^{5}, c_{22}=10^{4}$, $c_{12}=10^{3}, c_{66}=10^{3}, \varepsilon=0.1$, and $R_{e}=1$. In addition, we use a sequence of non-uniform meshes composed of $N=600 q, q \in\{1,4,16,64,256\}$, linear finite elements distributed in three intervals: $375 q$ elements in $0<R<0.07 R_{e}, 125 q$ elements in $0.07 R_{e}<R<0.46 R_{e}$, and $100 q$ elements in $0.46 R_{e}<R<R_{e}$.

To obtain the approximations for the constants of integration, we first isolate $C_{i}, i=1,2,3,5,7$, in $(21),(23)$, and (26). We then replace $u_{r}$ and $u_{\theta}$ by their respective approximations $u_{r}^{N}$ and $u_{\theta}^{N}$. The resulting expressions are given by

$$
\begin{aligned}
& \hat{C}_{1}^{N}(R)=u_{r}^{N} / R^{k}, \quad \hat{C}_{2}^{N}(R)=u_{\theta}^{N} / R, \quad \hat{C}_{3}^{N}(R)=\frac{u_{r}^{N}-\bar{u}_{r}\left(R_{e} / R\right)^{k}}{R^{k}-\left(R_{e}^{2} / R\right)^{k}}, \\
& \hat{C}_{5}^{N}(R)=\frac{u_{\theta}^{N}}{R-R_{e}^{2} / R}, \quad \hat{C}_{7}^{N}(R)=\left(R+u_{r}^{N}\right)^{2}+\left(u_{\theta}^{N}\right)^{2}-\varepsilon R^{2} .
\end{aligned}
$$

In Figure 1 we show curves of $\hat{C}_{i}^{N}(R), i=1,2,3,5,7$, given by (27), where the limits of the intervals of each curve are determined from numerical approximations of $R_{a}$ and $R_{b}$ obtained from the finite element simulation. These values are given by, respectively, $R_{a}^{N}=0.002$ and $R_{b}^{N}=0.019$. In Figures 1a and 1b we show $\hat{C}_{1}^{N}(R)$ and $\hat{C}_{2}^{N}(R)$, respectively, versus $R$ in the interval $(0,0.003)$. In Figures $1 \mathrm{c}$ and $1 \mathrm{~d}$ we show $\hat{C}_{3}^{N}(R)$ and $\hat{C}_{5}^{N}(R)$, respectively, versus $R$ in the interval $(0,1)$, and in Figure 1e we show $\hat{C}_{7}^{N}(R)$ versus $R$ in the interval $(0,0.02)$. Except for Figure 1a, we see from 


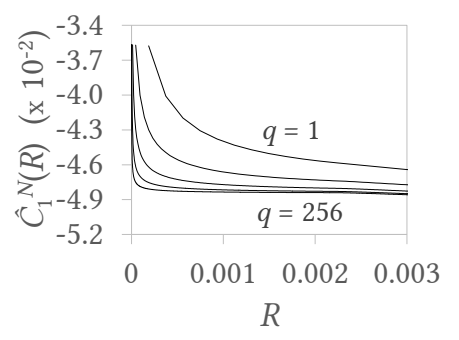

(a) $\hat{C}_{1}^{N}(R)$

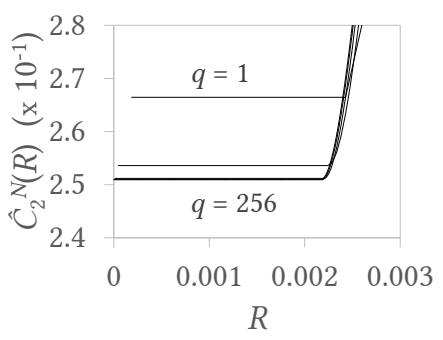

(b) $\hat{C}_{2}^{N}(R)$

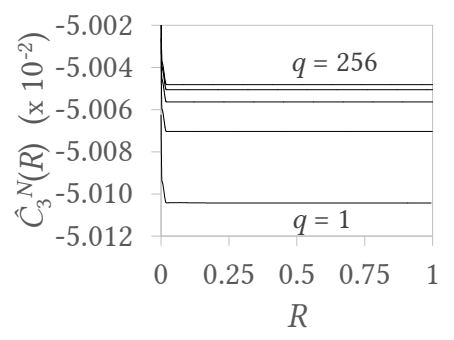

(c) $\hat{C}_{3}^{N}(R)$

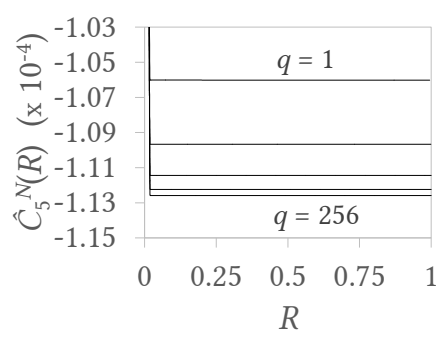

(d) $\hat{C}_{5}^{N}(R)$

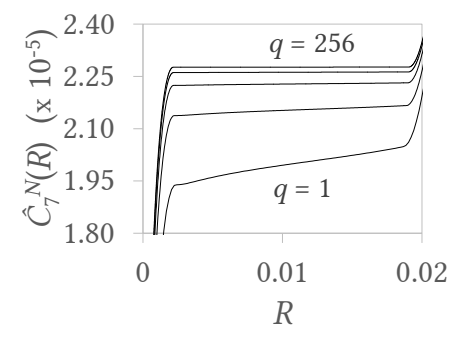

(e) $\hat{C}_{7}^{N}(R)$

Figure 1: $\hat{C}_{i}^{N}(R), i=1,2,3,5,7$, versus radius $R$ using the numerical solutions obtained with the meshes parameterized by $q=1,4,16,64,256$.

these figures that, in the intervals where the associated constants of integration $C_{i}$ are defined, the functions $\hat{C}_{i}^{N}(R)$ converge to limit functions that are constant as $q$ and, consequently, $N$ increases. In the case of Figure 1a, we see that $\hat{C}_{1}^{N}(R)$ is nearly constant outside a small neighborhood of the origin and that the size of this neighborhood tends to zero as $N$ increases. This is due to the singular behavior of the radial displacement (21a) at the origin, since $k<1$.

The convergence results observed in Figure 1 indicate that there is very good agreement between analytical and numerical results, allowing us to use the numerical results to determine the approximate values of the constants of integration, which we denote as $C_{i}^{N}, i=1,2,3,5,7$. We take $C_{i}^{N}$ as the mean value of the function $\hat{C}_{i}^{N}(R)$ evaluated at certain mesh nodes, as follows,

$$
\begin{array}{rlrl}
C_{1}^{N} & :=\frac{1}{M_{>}^{a}} \sum_{R_{i} \in B_{>}^{a}} \hat{C}_{1}^{N}\left(R_{i}\right), & C_{2}^{N}:=\frac{1}{M_{>}^{a}} \sum_{R_{i} \in B_{>}^{a}} \hat{C}_{2}^{N}\left(R_{i}\right), \quad C_{3}^{N}:=\frac{1}{M_{>}^{b}} \sum_{R_{i} \in B_{>}^{b}} \hat{C}_{3}^{N}\left(R_{i}\right), \\
C_{5}^{N}:=\frac{1}{M_{>}^{b}} \sum_{R_{i} \in B_{>}^{b}} \hat{C}_{5}^{N}\left(R_{i}\right), & C_{7}^{N}:=\frac{1}{M_{-}} \sum_{R_{i} \in B_{=}} \hat{C}_{7}^{N}\left(R_{i}\right),
\end{array}
$$

where $R_{i}$ is the position of the $i$-th mesh node and $M_{>}^{a}, M_{=}$, and $M_{>}^{b}$ are the number of mesh nodes in the intervals $B_{>}^{a}:=\left(0, R_{a}^{N}\right), B_{=}:=\left(R_{a}^{N}, R_{b}^{N}\right)$, and $B_{>}^{b}:=\left(R_{b}^{N}, R_{e}\right)$, respectively. Using our most refined mesh, which is associated with $q=256$, expressions (28) yield

$$
\begin{aligned}
& C_{1}^{N}=-4.826 \times 10^{-2}, \quad C_{2}^{N}=2.510 \times 10^{-1}, \quad C_{3}^{N}=-5.005 \times 10^{-2}, \\
& C_{5}^{N}=-1.126 \times 10^{-4}, \quad C_{7}^{N}=2.277 \times 10^{-5} .
\end{aligned}
$$

To determine the radii $R_{a}$ and $R_{b}$ of the annular region $\mathcal{B}_{=}$, we evaluate (26) at $R=R_{a}$ using the expressions in (21) and at $R=R_{b}$ using the expressions in (23). The constants of integration 
in the resulting expressions are substituted by their approximations $C_{i}^{N}, i=1,2,3,5,7$, yielding

$$
\begin{aligned}
& \left(C_{1}^{N} R_{a}^{k}+R_{a}\right)^{2}+C_{2}^{N} R_{a}^{2}-\varepsilon R_{a}^{2}-C_{7}^{N}=0 \\
& {\left[C_{3}^{N} R_{b}^{k}+R_{b}+\left(\bar{u}_{r} R_{e}^{k}-C_{3}^{N} R_{e}^{2 k}\right) / R_{b}^{k}\right]^{2}+\left[C_{5}^{N}\left(R_{b}-R_{e}^{2} / R_{b}\right)\right]^{2}-\varepsilon R_{b}^{2}-C_{7}^{N}=0 .}
\end{aligned}
$$

Using parameter values introduced above together with (29) and solving numerically the equations (30a) and (30b) for $R_{a}$ and $R_{b}$, respectively, we obtain $R_{a}^{A}=2.153 \times 10^{-3}$ and $R_{b}^{A}=1.906 \times 10^{-2}$, which are very close to the approximations $R_{a}^{N}=0.002$ and $R_{b}^{N}=0.019$ obtained from the finite element simulation.

\section{Conclusions}

We have investigated the problem of a cylindrically anisotropic disk in equilibrium with no body force, which is subjected to a prescribed displacement along its external boundary. We have investigated this problem in the context of a constrained minimization theory of elasticity and determined closed-form expressions for a rotationally symmetric solution, having the form (18), which are valid in $\mathcal{B}_{>}$. In $\mathcal{B}_{=}$, we have used the local injectivity constraint to find a nonlinear relation between the displacement components. The expressions depend on constants of integration that are determined numerically using finite element approximations of the rotationally symmetric solution. We have also determined the inner and outer radius of $\mathcal{B}_{=}$, which is an annular region. The results presented in this work are in good agreement with the finite element approximations of the rotationally symmetric solution obtained in our previous work [2].

\section{Acknowledgments}

The authors acknowledge the support of National Council for Scientific and Technological Development (CNPq), grant $\mathrm{n}^{\circ}$ 420099/2018-2, and Coordination for the Improvement of Higher Education Personnel (CAPES) - Finance Code 001.

\section{References}

[1] Aguiar, A. R. Local and global injective solution of the rotationally symmetric sphere problem, Journal of Elasticity, 84:99-129, 2006. DOI: 10.1007/s10659-006-9058-0.

[2] Aguiar, A. R. and Rocha, L. A. Numerical investigation of bifurcation instability in constrained minimization problem of elasticity, Proceedings of the Ibero-Latin-American Congress on Computational Methods in Engineering, 2020. ISSN: 2675-6269.

[3] Christensen, R. M. Properties of carbon fibers, Journal of the Mechanics and Physics of Solids, 42:681-695, 1994. DOI: 10.1016/0022-5096(94)90058-2.

[4] Fosdick, R., Freddi, F. and Royer-Carfagni, G. Bifurcation instability in linear elasticity with the constraint of local injectivity, Journal of Elasticity, 90:99-126, 2008. DOI: 10.1007/s10659007-9134-0.

[5] Fosdick, R. and Royer-Carfagni, G. The constraint of local injectivity in linear elasticity theory, Proceedings of the Royal Society A: Mathematical, Physical and Engineering Sciences, 457:2167-2187, 2001. DOI: 10.1098/rspa.2001.0812. 